\title{
Diversidad de Pequeños Roedores en una selva baja caducifolia espinosa del noreste de Yucatán, México
}

\author{
José A. Cimé-Pool', Silvia F. Hernández-Betancourt ${ }^{2 *}$, \\ Roberto C. Barrientos ${ }^{3}$ y Alejandro A. Castro-Luna ${ }^{4}$
}

\section{Abstract}

The aim of this study was to compare the diversity of small rodents along four unequally disturbed habitats in a low deciduous forest in Dzilam de Bravo, Yucatan: an abandoned pastureland (AP) with five-year old (SCA), ten-year old (SDA), and more than ten-year old (SMD) forests. A total of 478 rodents were captured with 1,754 recaptures from May 2005 to April 2006. They were included in six species and two families. Our work was done in two grids of 5,000 $\mathrm{m}^{2}$ with a distance of $10 \mathrm{~m}$ between rows and columns; Sherman's traps were set two nights per month in all sites. The habitat with the highest diversity $\left(H^{\prime}=1.328\right)$ and specific richness was the site with the highest disturbance and the lowest diversity $\left(H^{\prime}=0.6321\right)(\mathrm{PA})$, but the highest abundance was in the SMD forest. Differences in diversity among sites were significant $(P<0.05)$, except between SCA and SDA forests. The abundance values differed strongly in all sites. The abundance patterns of $O$. phyllotis were influenced by a disturbance gradient due to human influence and topography of the area, factors associated to the diversity observed.

Key words: Diversity, abundance, disturbance gradient, small rodents, low deciduous forest, Yucatan, Mexico.

\section{Resumen}

De mayo de 2005 a abril de 2006 se monitorearon pequeños roedores en cuatro sitios que representaron un gradiente de perturbación en Dzilam de Bravo y localidades aledañas: Pastizal abandonado (PA), selva baja caducifolia de cinco años (SCA), selva de hasta diez años (SDA) y selva de más de diez años de edad (SMD). El objetivo fue comparar la diversidad de ratones a través de este gradiente. Se implementaron dos cuadrantes de 5,000 $\mathrm{m}^{2} / \mathrm{sitio}$, usando 50 trampas Sherman en una cuadrícula equidistante de 10 $\mathrm{m}$ durante dos noches consecutivas por mes en cada sitio. Se capturaron 478 ratones de seis especies con 1,754 recapturas. La SMD aportó el 38\% de las capturas mientras que PA el $16 \%$. Ototylomys phyllotis y Peromyscus yucatanicus aportaron el $47 \%$ y el

\footnotetext{
${ }^{1}$ Departamento de Impacto y Riesgo Ambiental, SEMARNAT Delegación Yucatán. Av. Pérez Ponce No. 110, Col. Itzimná, Mérida, Yucatán, CP 97100, E-mail: jose.cime@semarnat.gob.mx

${ }^{2}$ Departamento de Zoología, Campus de Ciencias Biológicas y Agropecuarias, Universidad Autónoma de Yucatán. Carr. Mérida-Xmatkuil Km 15.5 AP 4-116 Itzimná, Mérida, Yucatán, CP 97000, E-mail: hbetanc@uady.mx, autor corresponsal

${ }^{3}$ Departamento de Ecología, Campus de Ciencias Biológicas y Agropecuarias, Universidad Autónoma de Yucatán. Carr. Mérida-Xmatkuil Km. 15.5 AP. 4-116 Itzimná, Mérida, Yucatán, CP 97000, E-mail: rcarlos@uady.mx

${ }^{4}$ Instituto de Ecología, A. C. Carretera Antigua a Coatepec No. 351, El Haya. AP 63. Xalapa, Veracruz, CP 91070. Email: castrolun@hotmail.com
} 
$41 \%$, respectivamente del total de las capturas. El sitio con la mayor riqueza específica y diversidad fue PA $\left(H^{\prime}=1.328\right)$, el sitio menos diverso fue SMD $\left(H^{\prime}=0.6321\right)$, pero con las máximas abundancias. Se presentaron diferencias significativas $(P<0.05)$ entre sitios en cuanto a la diversidad excepto entre la SCA con SDA. Los valores de abundancia difieren fuertemente en todos los sitios. Las abundancias de O. phyllotis se vieron afectadas por el gradiente de perturbación debido a la influencia antropogénica y la topografía que están asociados a la diversidad observada.

Palabras clave: Diversidad, abundancia relativa, gradiente de perturbación, pequeños roedores, selva baja caducifolia, Yucatán, México.

Introducción

Se conoce muy poco acerca del comportamiento y la distribución de las comunidades de pequeños roedores en mosaicos de vegetación con diferente grado de regeneración. En general, es muy poca la información del efecto de la perturbación sobre la diversidad de pequeños roedores y en especial en la selva baja (García-Estrada et al. 2002), que es el tipo más frecuente de vegetación en el estado de Yucatán.

Los pequeños mamíferos son componentes clave en los procesos de sucesión y regeneración de las selvas tropicales, debido a que juegan un papel importante en la depredación y postdispersión de semillas (Adler 1998; DeMattia et al. 2004, 2006). Algunas especies de pequeños roedores como los heterómidos dispersan especies de plantas pioneras de los sitios perturbados y en sus alrededores. Además, sus poblaciones son abundantes y sirven de base a la cadena trófica, manteniendo a algunos carnívoros primarios como reptiles, aves, y mamíferos medianos, por lo que su presencia así como las fluctuaciones en sus abundancias, se reflejan en las poblaciones de sus depredadores (Nupp y Swihart 1998). Es importante considerar su papel como reservorios de enfermedades como la de lime, leshmaniasis, hantavirus y leptospirosis (Chablé-Santos et al. 1995; Allan et al. 2003; Sánchez-Cordero et al. 2005). Algunas especies son susceptibles de convertirse en plaga cuando sus hábitats son transformados en enormes extensiones de cultivos y la explotación del nuevo hábitat les resulta favorable. Desde esta óptica los pequeños roedores pueden ser indicadores ecológicos, debido a que los cambios en sus abundancias y diversidad pueden reflejar modificaciones en el hábitat.

Una manera de medir la alteración por el uso de los sistemas naturales, es comparando la composición y abundancia de las comunidades de roedores en sitios con vegetación madura, en recuperación y en sistemas productivos (August 1983; Mellink 1985; Horváth et al. 2001; Riojas-López, 2006). Se ha documentado que los sitios perturbados determinan la abundancia y diversidad de pequeños roedores debido a que son complejos y heterogéneos en espacio y tiempo (Horváth et al. 2001; Vera y Conde y Rocha 2006). Nuestros objetivos fueron evaluar y comparar la diversidad de pequeños roedores en un gradiente de perturbación de selva baja caducifolia en el norte del estado de Yucatán, México, y determinar la asociación entre la abundancia relativa de los pequeños roedores y los atributos de la vegetación. 


\section{Material y métodos}

\section{Área de estudio.}

El estudio se realizó en Dzilam de Bravo, Yucatán donde se encuentra la Reserva de Dzilam, los sitios de muestreo estatal (Fig. 1). La vegetación del área II, III y IV se ubicaron fuera de ella y el I dentro del estudio corresponde en general a una selva baja caducifolia espinosa (SBCE) que contiene 386 especies vegetales, incluidas en

Figura 1. Mapa del área de estudio, con los sitios de muestreo, tres en la periferia (SDA, SCA y PA) y uno (SMDA) dentro de la Reserva Estatal de Dzilam, Yucatán, México.
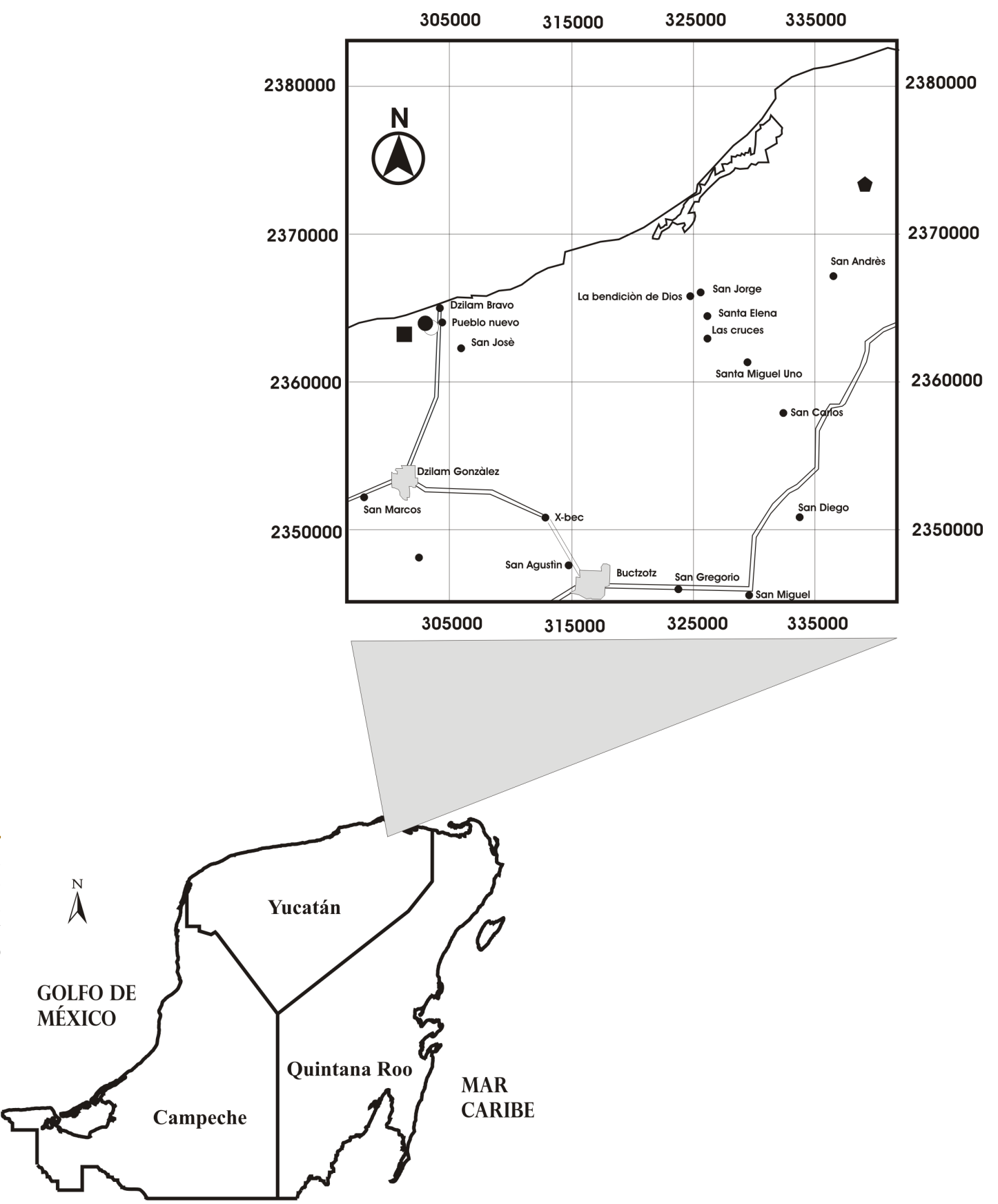
295 géneros y 96 familias botánicas. Las familias más representativas son: Fabaceae, Poaceae, Euphorbiaceae, Asteraceae y Cyperaceae (Flores y Espejel 1994; Gobierno del Estado de Yucatán 2006; Ortíz-Díaz et al. 2008). Para la selva baja caducifolia presente en los sitios y en un área de 1000 m², Escárraga-Paredes (2009) registró 195 géneros y 124 especies vegetales. Entre las especies dominantes se encuentran: Cnidoscolus aconitifolius, Plumeria rubra, Acanthocereus pentagonus, Bursera simaruba, Aphelandra deppeana, Anthurium schlechtendalii, Neea psychotrioides, Aechmea bracteata, Metopium browneii, Opuntia stricta, Beucarnea pliabilis, Psidium sartotianum var. yucatanense, Jatropha gaumeri, Commelina erecta, Gymnopodium floribundum, Selenicereus donkelaarii y Talisia olivaeformis, siendo la familia más diversa la Fabaceae (Leirana-Alcocer et al. 2009).

El clima del área es cálido-seco, intermedio entre los climas de tipo árido y húmedo, caracterizado por lluvias escasas. La temperatura promedio anual es de 25.5 ${ }^{\circ} \mathrm{C}$, con precipitación promedio anual de 970 mm y evaporación de 1800 mm (García 1988). El régimen de lluvias se divide en una época seca, de noviembre a abril y una época de lluvias, de mayo a octubre (Duch 1988).

\section{Diseño del muestreo y captura de pequeños roedores}

Sitios de muestreo.- Se seleccionaron cuatro sitios (en propiedad privada) con diferente grado de perturbación tipificados por medio del análisis de las variables de la estructura de la vegetación (e. g. altura, cobertura, diámetro a la altura del pecho (DAP) y dominancia). El promedio de las variables se indican en la Tabla 1.

\begin{tabular}{lllll}
\hline Variables & SMD & SDA & SCA & PA \\
\hline Especies arbóreas & 28 & 28 & 18 & 0 \\
DAP arbóreas $(\mathrm{cm})$ & 8.5 & 8.87 & 7.03 & 0 \\
Área basal arbóreas $(\mathrm{m})$ & 26.7 & 27.85 & 10.6 & 0 \\
Cobertura arbóreas $(\mathrm{m})$ & 4.3 & 4.33 & 3.38 & 0 \\
Altura arbóreas $(\mathrm{m})$ & 8.24 & 4.84 & 2.37 & 0 \\
Especies arbustivas & 23 & 31 & 17 & 18 \\
Cobertura arbustivas $(\mathrm{cm})$ & 10.26 & 94.88 & 128.41 & 209.9 \\
Especies herbáceas & 22 & 32 & 11 & 48 \\
Cobertura herbáceas $(\mathrm{cm})$ & 101.32 & 80.78 & 101.42 & 95.38 \\
\hline
\end{tabular}

Tabla 1: Atributos de la vegetación utilizados para la tipificación y selección de los sitios de monitoreo y la correlación de las abundancias de pequeños roedores. $\mathrm{SMD}=$ Selva de más de diez años, SDA $=$ Selva de hasta diez años, $\mathrm{SCA}=$ Selva de hasta cinco años, PA= Pastizal abandonado .

Sitio I.- Corresponde a una selva baja caducifolia espinosa (SBCE) con más de 10 años (SMD) de recuperación, ubicado en el Rancho San Salvador (UTM Zona 16: X= $339060.9529, \mathrm{Y}=2373303.088$ ). El rancho tiene una superficie de $890 \mathrm{ha}$, de las cuales el $25 \%$ son pastizales inducidos y el $75 \%$ selva en diversas fases de regeneración. El sitio es poco frecuentado debido al difícil acceso. La distancia que separa SMD con el sitio II es de $40.5 \mathrm{~km}$ (Fig. 1). El suelo es pedregoso y propenso a inundaciones. El área de muestreo tiene una superficie de aproximadamente 30 ha. Presenta los estratos herbáceo, arbustivo y arbóreo, este último con alturas de nueve a $13 \mathrm{~m}$.

Sitio II.- Está ubicado dentro del Rancho San Gregorio (UTM Zona 16: X= 300730.1093, 
$Y=2363924.897)$. Es un fragmento de SBCE de seis hectáreas de hasta 10 años de recuperación (SDA). En este fragmento se realiza aprovechamiento maderable para la construcción de cercos. Está rodeado principalmente por pastizales y agroecosistemas tales como cultivos de papaya, chile habanero y en menor escala maíz por lo que es común el uso de herbicidas. Este fragmento está unido a otros por una delgada franja de vegetación (tolché), que delimita un potrero de otro. En terrenos contiguos se produce ganado. Existe un camino de acceso al sitio muy transitado por vehículos principalmente motorizados. La distancia de SDA con el sitio III es de $2.5 \mathrm{~km}$ (Fig. 1). Presenta tres estratos: herbáceo, arbustivo y arbóreo, siendo las alturas de las especies arbóreas entre cuatro a siete metros.

Sitio III.- Es un continuo de SBCE de hasta cinco años de recuperación (SCA) de aproximadamente 16 ha ubicado dentro del Rancho Santa Teresa (UTM Zona 16: X= 302531, $Y=2363871$ ) en el que no hay actividad productiva. El sitio está rodeado por pastizales y acahuales, y no existe vía de acceso, por lo que no hay afluencia vehicular. La distancia de SCA al sitio IV es de 0.6 km (Fig. 1). Presenta los estratos: herbáceo, arbustivo y arbóreo, siendo las alturas de las especies arbóreas entre 1 y 3.5 m.

Sitio IV.- Es un pastizal abandonado (PA) de ocho hectáreas ubicado dentro del Rancho Santa Teresa (UTM Zona 16: $X=302999, Y=2363716)$. Se encuentra a $3 \mathrm{~km}$ de Dzilam de Bravo. En el sitio no había habido pastoreo de ganado bovino aproximadamente desde año y medio antes del estudio. Durante el período de muestreo hubo control de la vegetación a través del corte de las hierbas y arbustos. Al igual que en SCA no existe vía de acceso para vehículos (Fig. 1). Se presentan sólo los estratos herbáceo y arbustivo. Las alturas de las especies arbóreas son entre los 1.03 a $1.81 \mathrm{~m}$.

Captura de pequeños roedores.- Los monitoreos se realizaron mensualmente desde mayo de 2005 hasta abril de 2006, trampeando durante dos noches consecutivas cada sitio (ocho noches totales). En cada sitio se colocaron dos cuadrantes de 5,000 $\mathrm{m}^{2}$ cada uno considerando el efecto de borde. El establecimiento de dos cuadrantes fue con la finalidad de obtener registros en un área lo más amplia posible, así como una mayor captura de individuos. Cada cuadrante consistió de 10 filas y cinco columnas con intervalos de $10 \mathrm{~m}$ entre ellas, en total fueron 100 estaciones de trampeo por sitio, en cada una se colocó una trampa Sherman. Como cebo se utilizaron semillas de girasol (Helianthus annus). Se empleó el método de captura recaptura (Krebs 1985) y cada individuo se marcó por ectomización de falanges (Rudran 1996).

Análisis de datos. Debido a que los estimadores asintóticos consistentemente subestiman la riqueza de especies, se ha sugerido el uso de estimadores no paramétricos como la mejor opción (Colwell y Coddington 1994; Cao et al. 2004; Hortal et al. 2006). Se utilizaron los estimadores no paramétricos de Chao 2 y Jackknife de primer orden (Jack 1) para determinar el número de especies esperadas para cada sitio utilizando el paquete estadístico EstimateS 7.5 (Colwell 2005). El estimador Chao 2 se aplica para la distribución de las especies entre muestras y sólo requiere datos de presencia-ausencia. Se basa en el número de especies representadas en una y dos muestras (Colwell y Coddington 
1994). El estimador Jacknife de primer orden (Jack 1), disminuye la subestimación del verdadero número de especies en una comunidad, reduciendo el sesgo del orden $(1 / n)$ de los muestreos. Se basa en el número de especies representadas en una sola muestra (Colwell y Coddington 1994; Moreno 2000). Se recomienda el uso de estos estimadores cuando se tiene una unidad de esfuerzo pequeña, y para áreas pequeñas de muestreo (Escalante et al. 2002; Tøttrup et al. 2005; Hortal et al. 2006); aunque se ha comprobado que el estimador Chao 2 se ajusta mejor para muestras pequeñas, debido a que es menos sesgado al número de muestras (Colwell y Coddington 1994).

Para comparar los patrones de abundancia de especies entre sitios y especies se usó una gráfica de abundancia - diversidad o curva de Whitaker (Feinsinger 2001). Como indicadores de la diversidad de pequeños roedores y abundancias para cada sitio, se usaron las variables: 1) El número de especies de pequeños roedores. 2) La diversidad, medida con el índice de Shannon-Wiener $H^{\prime}$ empleando el paquete estadístico BIODIV (Baev y Penev 1995), el cual se basa en el número de especies raras (Magurran 2004), y 3) El porcentaje y abundancia total de las especies. Estas variables son comúnmente usadas como indicadores de la diversidad y estructura de una comunidad (Medellín et al. 2000). Las especies raras son aquellas que viviendo y reproduciéndose en el lugar de muestreo, están representadas por poblaciones con muy pocos individuos (Halfter y Moreno 2005). Además, se utilizó el índice de dominancia de Berger-Parker (d), que se basa en la importancia proporcional de las especies más abundantes. Es completamente independiente del número de especies (S), por lo que algunos autores lo recomiendan como uno de los mejores índices para calcular la dominancia (Moreno 2000) y el índice de equidad de Pielou (Magurran 2004).

Análisis estadísticos.- Para obtener las diferencias entre los índices de diversidad $\left(H^{\prime}\right)$ entre sitios se utilizó una prueba de " $t$ " modificada por Hutcheson (Zar 1999). Para determinar si había una correlación entre las abundancias de pequeños roedores con los atributos de la vegetación en cada sitio (e. g. altura, cobertura, DAP's y dominancia), se aplicó el coeficiente de intervalo de correlación de Spearman (Zar 1999). En todos los casos se utilizó un $\alpha=0.05$.

Resultados

Diversidad de pequeños roedores.- Se capturaron 478 individuos con 1,754 capturas, en 9,600 noches trampa. Estos correspondieron a seis especies de las familias Cricetidae y Heteromyidae (Tabla 2). El sitio que aportó el mayor número de individuos fue SMD con el 38\% (40\% recapturas), seguido de SDA con el 24\% (20\% recapturas), SCA con el $22 \%$ (24\% recapturas) y PA con el $16 \%$ (16\% recapturas). Ototylomys phyllotis fue la especie más abundante (47\% de las capturas), seguida por Peromyscus yucatanicus $(41 \%)$. Otras cuatro especies se consideraron especies raras, por presentar capturas menores al 10\% del total: Heteromys gaumeri, Reithrodontomys gracilis, Mus musculus y Sigmodon hispidus.

En SMD se capturó el 50\% de la riqueza total, en SDA el 83\%, en SCA el 67\% y en PA el $100 \%$. En cuanto a la riqueza acumulada por sitio, en SMD el total de especies se capturó con un esfuerzo de 1,800 noches trampa (noche 18), en SDA con 1,100 noches trampa (noche 11), en SCA con 1,700 noches trampa (noche 17) y en el PA con 
2,300 noches trampa (noche 23). En este último sitio, con un esfuerzo de captura de una noche se registró el $67 \%(n=4)$ y al lograr un esfuerzo de captura de 1,000 noches trampa se alcanzó el 83\% de las especies esperadas (Fig. 2). Con el esfuerzo de captura efectuado y considerando el estimador Jacknife 1, se capturó el 100\% de las especies esperadas para la SDA y SCA, el $75 \%$ para la SMD y el $86 \%$ para PA, mientras que con el estimador Chao 2 se capturó el 100\% para SMD, SDA y SCA de las especies esperadas y el $92 \%$ para PA. Por lo anterior consideramos adecuado el esfuerzo realizado.

\begin{abstract}
Tabla 2. Abundancia relativa y parámetros de diversidad de pequeños roedores en una selva baja caducifolia de Dzilam, al noreste de la Península de Yucatán, México.
\end{abstract}

$\mathrm{SMD}=$ Selva de más de diez años, SDA= Selva de hasta diez años, SCA $=$ Selva de cinco años, $\mathrm{PA}=$ Pastizal abandonado. * Endémica a la Península de Yucatán.

\begin{tabular}{llllll}
\hline & SMD & SDA & SCA & PA & TOTAL \\
\hline Familia Muridae & & & & & \\
Mus musculus & 0 & 6 & 0 & 4 & 10 \\
Peromyscus yucatanicus* & 52 & 61 & 54 & 30 & 197 \\
Ototylomys phyllotis & 128 & 44 & 26 & 25 & 223 \\
Reithrodontomys gracilis & 0 & 3 & 4 & 17 & 24 \\
Sigmodon hispidus & 0 & 0 & 0 & 1 & 1 \\
Familia Heteromyidae & & & & & \\
Heteromys gaumeri* & 1 & 2 & 19 & 1 & 23 \\
Abundancia relativa & 181 & 116 & 103 & 78 & 478 \\
Riqueza & 3 & 5 & 4 & 6 & 6 \\
H' $^{*}$ & 0.6321 & 1.023 & 1.124 & 1.328 & --- \\
Dominancia Berger-Parker & 0.7072 & 0.5259 & 0.5243 & 0.3846 & --- \\
Equitatividad J (Sp Max.) & 0.3528 & 0.5712 & 0.6273 & 0.7413 & --- \\
\hline
\end{tabular}

En cuanto a las gráficas de abundancia - diversidad, se observan diferencias entre las pendientes de la selva de más de diez años y el pastizal (Fig. 3). En la primera hay una mayor abundancia total, menor riqueza de especies y mayor dominancia, mientras que en PA hay una menor abundancia total, una mayor riqueza específica y una menor dominancia. Las selvas de SDA y SCA ocupan una posición intermedia entre estos dos extremos. P. yucatanicus y $O$. phyllotis se presentaron en la misma posición jerárquica en SDA, SCA y PA como primera y segunda especies más abundantes, respectivamente. La rata arborícola $O$. phyllotis fue la más abundante en SMD. Entre las especies raras únicamente $S$. hispidus se presentó en PA, las otras fueron compartidas en dos o tres sitios.

El sitio más diverso fue PA con un valor $H^{\prime}=1.328$; en contraste, el menos diverso fue SMD con un $H^{\prime}=0.6321$ (Tabla 2). La equidad aumentó del sitio poco perturbado al más perturbado. Heteromys gaumeri fue una especie única para SMD y PA y duplicada para SDA. Todas las especies son compartidas excepto S. hispidus que es una especie única y exclusiva para PA, esta especie se capturó en la penúltima noche de muestreo. Sólo se encontraron diferencias significativas entre la diversidad de PA y SCA $(t=2.03, g . I .=247, P<0.05)$, PA con SDA $(t=2.8, g . I .=209, P<0.05)$, $\mathrm{PA}$ con SMD $(t=7.79$, g. $I .=304, P<0.05)$, SCA con SMD $(t=6.71, g . l .=373, P<$ 


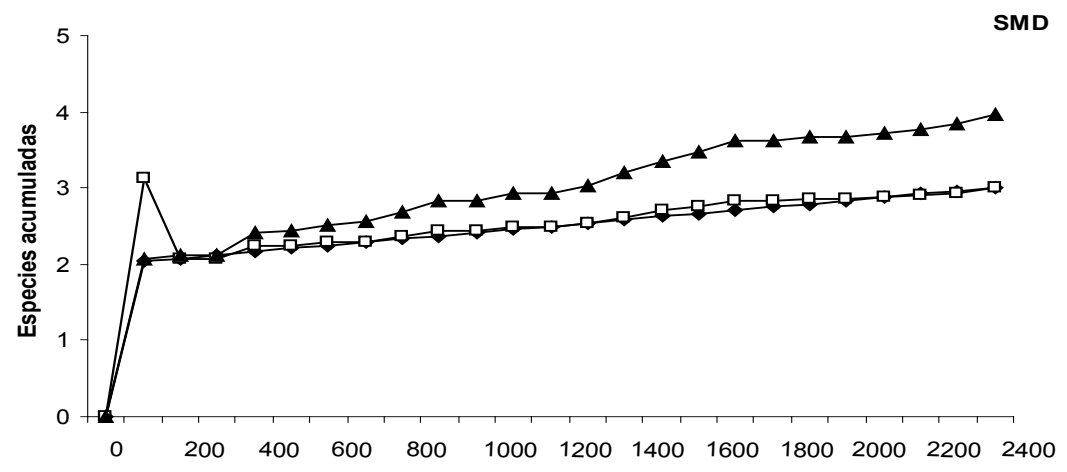

SDA
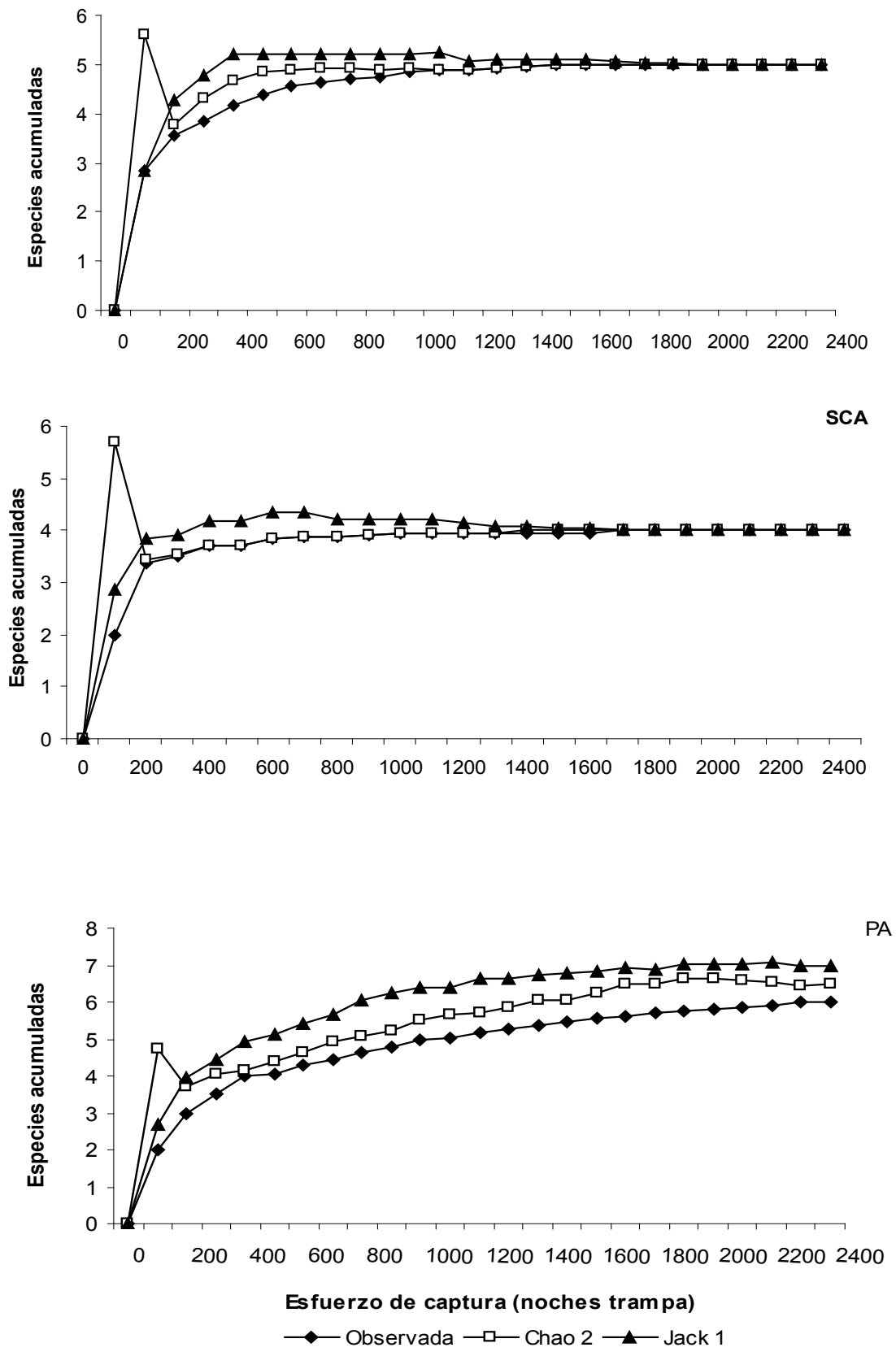

Figura 2. Curva de acumulación de especies de pequeños roedores en una selva baja caducifolia de Dzilam, al noreste de la Península de Yucatán, México. SMD= Selva de más de diez años, SDA= Selva de hasta diez años, $\mathrm{SCA}=$ Selva de hasta cinco años y $\mathrm{PA}=$ Pastizal abandonado. 
0.05) y SDA con SMD $(t=4.66, g . I .=439, P<0.05)$. Lo anterior indica que existe una gran heterogeneidad en la diversidad de roedores entre los sitios estudiados.

Relación entre la abundancia de roedores y los atributos de la vegetación.- La abundancia de $M$. musculus se relaciona inversamente con el porcentaje de cobertura de especies herbáceas $\left(r_{s}=-0.95, P<0.05\right)$. De manera similar la abundancia de $R$. gracilis se relaciona inversamente con a la altura $\left(r_{s}=-1.0, P<0.01\right)$ y al número de especies arbóreas $\left(r_{s}=-0.95, P<0.05\right)$. En contraste, la abundancia de $O$. phyllotis se relaciona directamente con la altura de los árboles $\left(r_{\mathrm{s}}=1.0, P<0.01\right)$, y al número de especies arbóreas $\left(r_{\mathrm{s}}=0.95, P<0.05\right)$. No se encontró ninguna relación en la abundancia de $P$. yucatanicus con los atributos de la vegetación $(P>0.05)$. Las abundancias de $O$. phyllotis fueron mayores en los sitios de mayor regeneración vegetal (SMD, SDA) que en los que la tuvieron menos (SCA, PA). Las máximas abundancias fueron en los meses de octubre en SMD y de enero-febrero en SDA, SCA y PA, aunque en los dos últimos sitios se observó otro máximo en septiembre-octubre de 2006. P. yucatanicus fue la especie más abundante en los sitios SDA, SCA y PA, presentando un máximo en la época seca (enero-febrero, Fig. 4).

\footnotetext{
Figura $\quad 3 . \quad$ Curva intervalo-abundancia de especies de pequeños roedores en una selva baja caducifolia de Dzilam, al noreste de la Península de Yucatán, México. $\mathrm{SMD}=$ Selva de más de diez años, $\mathrm{SDA}=$ Selva de hasta diez años, SCA= Selva de hasta cinco años y $P A=$ Pastizal abandonado. N = número de individuos de todas las especies, ni $=$ número de individuos de cada especie.
}

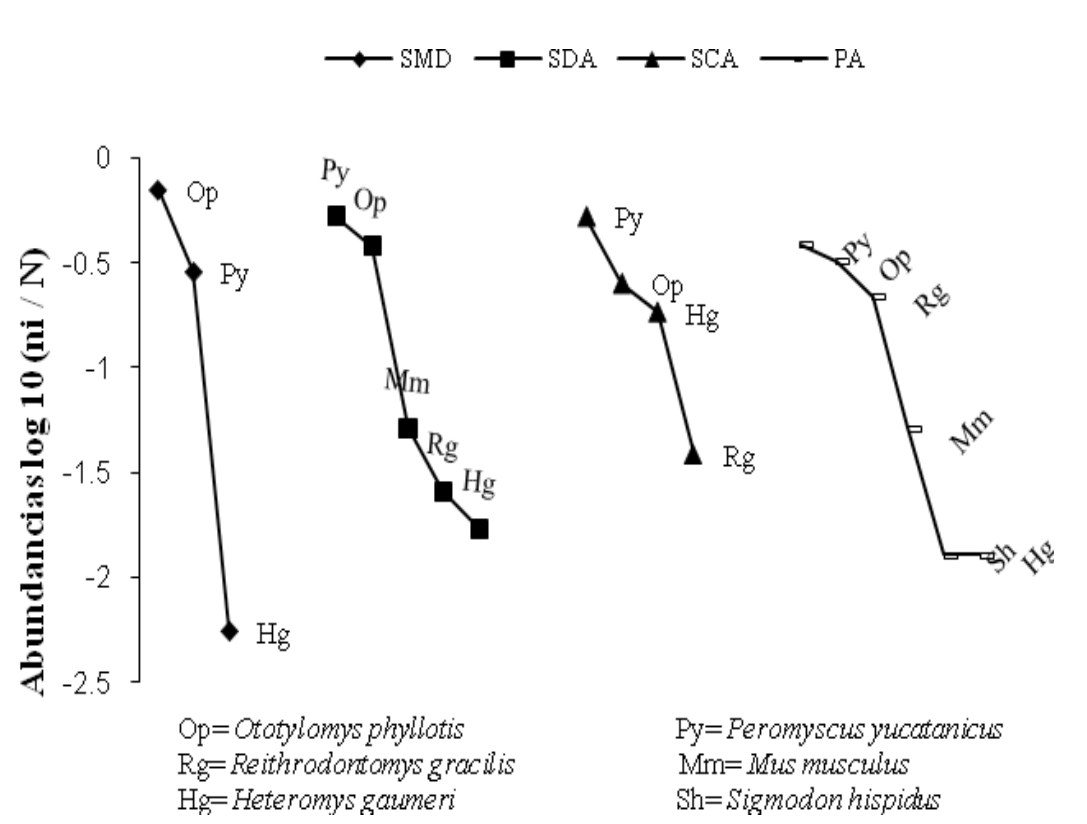

\section{Discusión}

Riqueza y diversidad.- El número de especies observadas representa el $75 \%$ de las registradas para la Reserva Estatal de Dzilam (Hernández-Betancourt et al. 1996) y el $50 \%$ de las descritas para el estado de Yucatán (Jones et al. 1974). La riqueza y diversidad de roedores (3-6 especies) fueron diferentes entre los sitios estudiados, presentándose una mayor diversidad en el sitio más perturbado (PA) con respecto al menos perturbado (SMD). Esto se atribuye probablemente a que es un área pequeña (8 ha) rodeada por 

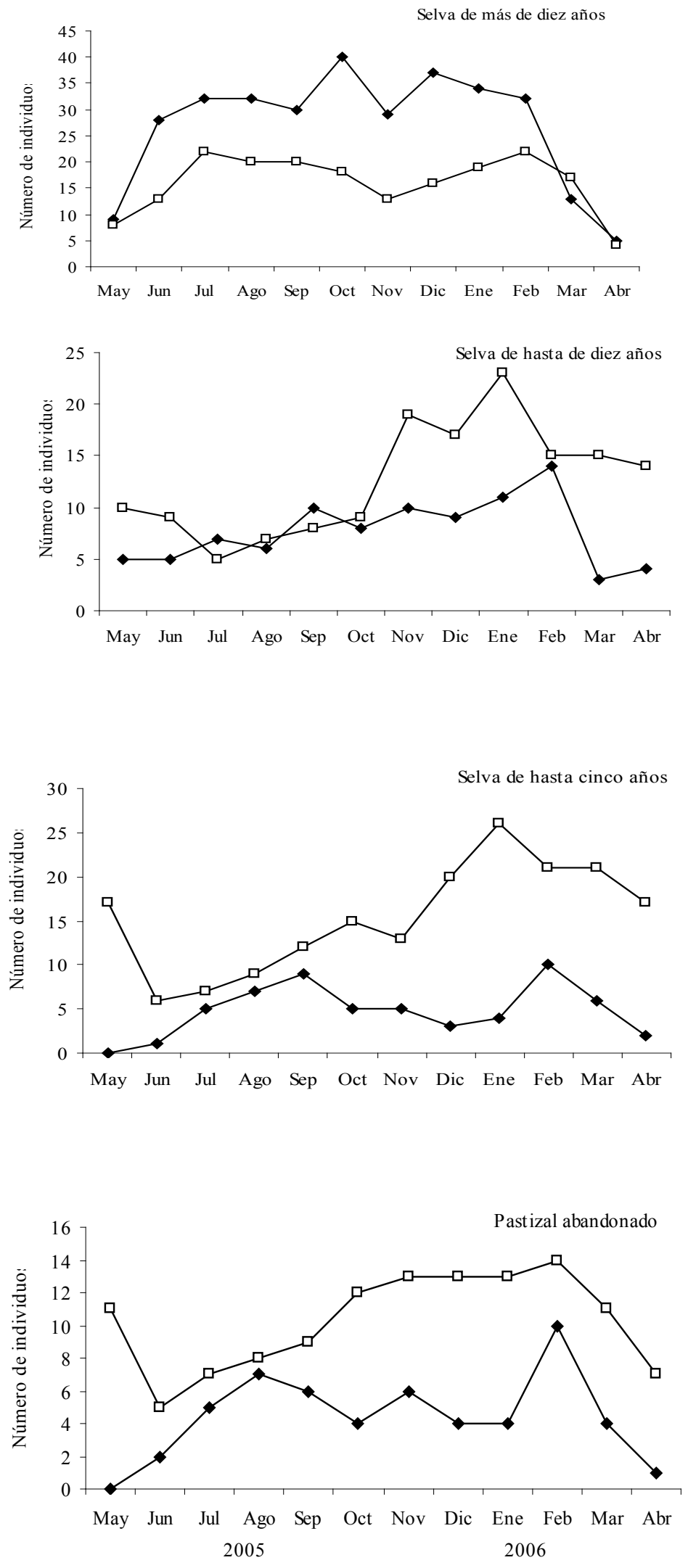

$\longrightarrow$ O. phyllotis $\square-$ P. yucatanicus

Figura 4. Fluctuaciones de las abundancias de Ototylomys phyllotis y Peromyscus yucatanicus en una selva baja caducifolia de Dzilam, al noreste de la Península de Yucatán, México. 
selva baja caducifolia espinosa en sucesión, lo que permitió capturar especies de hábitos arborícolas como O. phyllotis y la captura ocasional de H. gaumeri, así como a la presencia de M. musculus y $S$. hispidus que parecen preferir ambientes distintos a la selva.

Mus musculus es una especie introducida que se beneficia con la alteración del hábitat y las actividades antropogénicas (Bolger et al. 1997). Esta podría ser la razón por lo que la especie estuvo asociada negativamente con la cobertura herbácea en este estudio. Es notorio que su captura se realizó en los sitios perturbados o cercanos a aquellos dedicados a la actividad agrícola (e. g. cultivos de maíz y papaya) y ganadera. En cuanto a $S$. hispidus es una rata que se ha reportado relacionada a pastizales, lo mismo que afectando cultivos de caña y arrozales, aunque también se ha registrado en selva baja espinosa asociada con cultivos y ambientes mixtos (Cameron y Spencer 1981; Ceballos y Oliva 2005).

En el presente estudio se observó una mayor riqueza de especies en el sistema vegetal más perturbado (PA), como se ha visto en general para los mamíferos, esto se debe al incremento de la heterogeneidad espacial (Mellink 1985; Horváth et al. 2001; Riojas-López 2006; Vera y Conde y Rocha 2006). En una selva brasileña ocurrió algo semejante en un gradiente de perturbación, la diversidad de pequeños mamíferos fue comparativamente más baja en la selva no perturbada mientras que los valores altos de abundancia, riqueza y diversidad se observaron en los sitios de selva con niveles de perturbación intermedia (Vera y Conde y Rocha 2006). En contraste, en una selva baja caducifolia de El Limón, Morelos, México la alteración del hábitat no modificó la riqueza ni la diversidad de especies de pequeños roedores (García-Estrada et al. 2002).

Se observó que la riqueza de especies es comúnmente baja en los sistemas vegetales de la Península de Yucatán, debido a que en promedio se capturaron cinco. Las más frecuentes, pero en diferentes proporciones fueron: P. yucatanicus, H. gaumeri y O. phyllotis. Probablemente las primeras dos, por ser especies endémicas, han evolucionado y se han adaptado a las condiciones de la selva baja caducifolia (sustrato, vegetación, régimen climático, Young y Jones 1983; Schmidt et al. 1989) de la Península de Yucatán. Los fragmentos de selva con desarrollo vegetal intermedio como SDA y SCA todavía pueden mantener estables sus poblaciones y la diversidad faunística presente. O. phyllotis, es otra especie que puede presentarse en sitios perturbados aunque su abundancia se ve afectada como se observó en PA y SCA.

La riqueza registrada en este trabajo (seis especies) es semejante a la reportada en estudios realizados en áreas de selva baja caducifolia y selva mediana subcaducifolia de Reservas de los estados de Yucatán y Quintana Roo, México, que se encuentra entre cinco y ocho especies (Hernández-Betancourt et al. 1996, 2008a, b; Pozo y Escobedo 1999; Cimé-Pool et al. 2006). Tampoco hay grandes diferencias cuando se compara con agroecosistemas donde se han registrado entre cuatro y siete especies de roedores (Chablé-Santos et al. 1995; Cimé-Pool et al. 2007).

Heteromys gaumeri y S. hispidus fueron "especies turistas" (Halfter y Moreno 2005) para SMD y PA, respectivamente. Heteromys gaumeri por sus hábitos terrestres y alimentación a base de frutos y semillas (Schmidt et al. 1989) no puede habitar permanentemente en SMD, debido a que el suelo de este sitio es pedregoso y propenso a inundaciones en la época de lluvias. En cuanto a la captura ocasional de esta especie 
en PA, probablemente se debió a un desplazamiento ocasional, ya que sólo se capturó un individuo en este sistema. El control de herbáceas y arbustivas (deshierbe) en PA pudo tener como resultado la baja captura de $S$. hispidus. En sistemas productivos se ha observado que las abundancias de $S$. hispidus son mayores en la época húmeda, cuando se incrementa la cobertura de herbáceas (Cameron y Spencer 1981; ChabléSantos et al. 1995), al igual que otros agroecosistemas tradicionales de México como los del altiplano de San Luis Potosí (Mellink 1991, 1995) y agroecosistemas abandonados como la milpa y henequenales de Yucatán (Cimé-Pool et al. 2007).

Relación entre la abundancia de roedores y los atributos de la vegetación.- En este estudio se observó un claro efecto del grado de perturbación sobre la abundancia de las especies de roedores quizá debido a características de la vegetación y el manejo antropogénico realizado en cada sitio. Factores como el aislamiento de los fragmentos, tamaño, calidad y diversidad del parche, así como la cobertura vegetal, pueden determinar en gran medida la abundancia de las especies presentes en un sitio particular (Bolger et al. 1997, Arroyo-Rodríguez y Mandujano 2009).

La abundancia de M. musculus fue del 5\% de la captura total en PA y SDA, y su presencia se relacionó inversamente al porcentaje de cobertura de especies herbáceas. En PA se tuvo una alta proporción de herbáceas y no hubo manejo constante, sólo deshierbes esporádicos. En contraste, en SDA se presentó menor porcentaje de herbáceas y un mayor número de claros por la extracción de madera, las actividades agrícolas y ganaderas que se realizaban en los terrenos aledaños, por lo tanto la abundancia de esta especie fue ligeramente mayor.

La abundancia de $R$. gracilis se relacionó inversamente a la altura y al número de especies arbóreas, probablemente debido a que esta especie prefiere sitios abiertos y secos (Young y Jones 1984). Características que se presentaron en PA. Por lo general, presenta abundancias bajas en otras localidades del estado como Molas y Dzibichaltún, donde hemos capturado pocos individuos, coincidiendo sus máximas capturas con los meses más secos. En un estudio en varias asociaciones vegetales de la Reserva de la Biosfera Ría Celestún, sólo se capturó en vegetación de duna costera con porcentajes de captura total menores al 10\% (Cimé-Pool et al. 2006).

El gradiente de sucesión vegetal impactó directamente sobre las abundancias relativas de $O$. phyllotis, así mismo, se observó que sus abundancias se relacionan directamente con la altura y número de especies vegetales arbóreas. En este estudio el sitio SMD fue el que cumplió estás condiciones. Esta rata es una especie arborícola de alimentación herbívoro-frugívoro que habita tanto selvas primarias como secundarias (Lawlor 1982), presentando las mayores abundancias en sitios con un estrato arbóreo bien desarrollado (Hernández-Betancourt et al. 2008a). El sitio SMD se inunda durante la época de lluvias por lo que sus abundancias fueron mayores en comparación con $P$. yucatanicus que es una especie semiarborícola (Young y Jones 1983; Reid 1997; Ceballos y Oliva 2005). Lo anterior se reafirma debido a que ocasionalmente se colocaron trampas a diferentes alturas (1.2 a $2.7 \mathrm{~m}$ ) obteniendo el 50\% de los individuos capturados entre 1.6 a $1.8 \mathrm{~m}$ como alturas más frecuentes.

El porcentaje de pedregosidad, pudo ser otro factor que influyera en la mayor abundancia de $O$. phyllotis, debido a que las mayores capturas $(70 \%$ de las capturas 
totales) se realizaron en SMD. En estaciones de trampeo situadas en pedregales con cobertura vegetal con árboles, arbustos y herbáceas y con presencia de lianas. En monitoreos de pequeños roedores en otras localidades del estado de Yucatán como Dzibilchaltún (Meneses 2008) y Lagunas de Yalahau (Miranda 2010) también se han observado mayores capturas de $O$. phyllotis en sitios con montículos y alto porcentaje de rocas. Esta observación ya ha sido documentada en localidades de la Península de Yucatán y Centroamérica (Reid 2009; Sáenz 1999).

El hecho de que no se haya observado una relación en las abundancias y los atributos de la vegetación para $P$. yucatanicus, probablemente se deba a que presenta una alta capacidad de habitar cualquier ambiente ya que es una especie omnívora y de reproducción contínua (Young y Jones 1983). En este estudio se observaron hembras reproductivas (preñadas y receptivas) durante todo el año. En contraste, en una asociación de bosque espinoso, manglar y palmas de coco (Cocos nucifera) en Playa de Oro, Colima, México, P. perfulvus especie endémica semiarborícola prefirió sitios con árboles cercanos, con vegetación del estrato inferior escasa, la del estrato superior densa y con poca hojarasca (Schnell et al. 2008).

En un Petén de Celestún, Yucatán $P$. yucatanicus fue la especie más abundante (59\%) y en una asociación de selva baja caducifolia con selva baja caducifolia inundable fue la única presente (Cimé-Pool et al. 2006). En contraste en la selva mediana subcaducifolia del estado de Yucatán se presenta en porcentajes bajos $(0.6 \%$ y el 2\%) en las capturas totales (Hernández-Betancourt et al. 2008a, b). Probablemente las máximas abundancias en sitios inundables se deba a que siendo una especie semiarborícola tiene la capacidad de forrajear en el estrato arbóreo (Reid 1997; CiméPool et al. 2006).

Con el presente estudio se concluye que la selva baja caducifolia espinosa de Dzilam en diferentes estados de regeneración, mantiene la riqueza, diversidad y abundancia de un alto porcentaje de pequeños roedores, principalmente los sitios de regeneración intermedia. Por lo tanto es necesario realizar estudios extensos en cuanto al efecto de los tamaños de los fragmentos y sobre la composición vegetal de los parches y compararlos con otros sistemas en regeneración, que incluyan sistemas agropecuarios en el área de estudio para tener un patrón más claro para conservar las comunidades de pequeños roedores que son un eslabón fundamental en el ecosistema selva del norte del estado de Yucatán.

\section{Agradecimientos}

Al sistema de becas PROMEP clave Promep/103.5/04/1416 UADY-EXB-64 y al proyecto CONACYT clave FOMIX YUC-2003-C02-043 por el financiamiento de esta investigación. A C. E. Moreno del Centro de Investigaciones Biológicas de la Universidad Autónoma del Estado de Hidalgo por la capacitación en el manejo de paquetes estadísticos sobre diversidad. Al Campus de Ciencias Biológicas y Agropecuarias Universidad Autónoma de Yucatán por las facilidades brindadas. A J. Leirana y L. Salinas del Departamento de Ecología realizaron los perfiles y análisis de la vegetación. A L. Ruiz Ruiz del Centro de Investigaciones Tropicales de Universidad Veracruzana y H. Estrada del CCBA-UADY por redigitalizar las imágenes. A los dueños de los predios por permitirnos el acceso a los sitios para realizar nuestra investigación. A S. Medina Peralta, 
J. M. Pech Canché, y dos revisores anónimos por sus sugerencias y comentarios. A E. López-Cobá, L. Guerrero-González, S. Cicero, C. Morales-Breck, A. Raymundo, A. Peña, M. López-Uribe, D. Lucero López, M. E. Estrella Martínez, L. Chulím, así como L. Aké y J. Cimé apoyaron en el trabajo de campo.

Referencias

Ader, G. H. 1998. Impacts of resource abundances of populations of a tropical forest rodent. Ecology 79:242-254.

Allan, B. F., F. Keesing, y R. S. Ostfeld. 2003. Effect of forest fragmentation of Lyme disease risk. Conservation Biology 17:267-272.

Arroyo-Rodríguez, V., y S. Mandujano. 2009. Conceptualization and measurement of habitat fragmentation from primates' perspective. Journal of Primatology 30:497-514.

August, P. 1983. The role of habitat complexity and heterogeneity in structuring tropical mammal communities. Ecology 64:1495-1507.

Baev, P. V., y L. D. Penev. 1995. BIODIV: Calculating biological diversity parameters, similarity, niche overlap and cluster analysis. PENSOFT, Sofia Moscow.

Bolger, D. T., A. C. Albert, R. M. Sauvajot, P. Potenza, C. Mccalvin, D. Tran, S. Mazzoni, y M. E. SoulÉ. 1997. Response of rodents to habitat fragmentation in coastal Southern California. Ecological Application 7:552-563.

Cameron, G. N., y S. R. Spencer. 1981. Sigmodon hispidus. Mammalian Species 158:1-9.

CaO, Y, D. P. Larsen, y D. White. 2004. Estimating regional species richness using a limited number of survey units. Ecoscience 11:23-35.

Ceballos, G., y G., Oliva. 2005. Los mamíferos silvestres de México. CONABiO - Fondo de Cultura Económica.

Chablé-Santos, J., N. Van Wynsberghe, S. Canto-lara, y F. Andrade. 1995. Isolation of Leishmania (I.) mexicana from wild rodents and their possible role on the transmission of localized cutaneus leishmaniasis in the State of Campeche, Mexico. American Journal of Tropical Medicine Higiene 53:141-145.

Cimé-Pool, J. A., J. B. Chablé-Santos, J. E. Sosa-Escalante, y S. F. Hernández-Betancourt. 2006. Quirópteros y pequeños roedores de la Reserva de la Biosfera Ría Celestún, Yucatán, México. Acta Zoológica Mexicana (n.s.) 22:127-131.

Cimé-Pool, J. A., S. F. Hernández-Betancourt, y J. B. Chablé-Santos. 2007. Comunidad de pequeños roedores en dos agroecosistemas de Yucatán, México. Revista Mexicana de Mastozoología 11:57-68.

Colwelt, R. K. 2005. EstimateS: Statistical estimation of species richness and shared species from simples. Versión7.5. Persistent URL < purl.oclc.org/estimates $>$.

Colwell, R. K., Y J. A. Coddington. 1994. Estimating terrestrial biodiversity through extrapolation. Philosophical Transaction: Biological Science, Great Britain 345:101-118.

Demattia, E. A., L. M. Curran, y B. J. Rathcke. 2004. Effects of small rodents and large mammals on Neotropical seeds. Ecology 85:2161-2170.

Demattia, E. A., B. J. Rathcke, L. M. Curra, R. Aguilar, y O. Vargas. 2006. Effect of small rodent and large mammals exclusion on seedling recruitment in Costa Rica. Biotropica 38:196-202. 
Duch, G. J. 1988. La conformación territorial del Estado de Yucatán. Los componentes del medio físico. Universidad Autónoma de Chapingo. Centro Regional de la Península de Yucatán.

Escalante, T., D. Espinosa, y J. J. Morrone. 2002. Patrones de distribución geográfica de los mamíferos terrestres de México. Acta Zoológica Mexicana (n. s.) 87:47-65.

Escárraga-Paredes, D. 2009. Estructura y composición de la selva baja caducifolia en las Reservas: Estatal del Palmar y Bocas de Dzilám, Yucatán. Tesis de licenciatura FMVZ- Universidad Autónoma de Yucatán.

Feinsinger, P. 2001. Designing field studies for biodiversity conservation. The Nature Conservancy and Island Press. Washington, D. C.

Flores, J. S., y I. EsPejel. 1994. Tipos de vegetación de la Península de Yucatán. Etnoflora Yucatanense. Fascículo 3, Universidad Autónoma de Yucatán.

García, E. 1988. Modificaciones al sistema de clasificación climática de Copen. $3^{\text {a }}$ Ed. Instituto de Geografía. Universidad Autónoma de México. México, D. F.

García-Estrada, C., M. L. Romero-Almaráz, y C. Sánchez-Hernández. 2002. Comparison of rodent communities in sites with different degrees of disturbance in deciduous forest of Southeastern Morelos, Mexico. Acta Zoológica Mexicana (n. s.) 85:153-168.

Gobierno Del Estado De Yucatán. 2006. Programa de manejo de la Reserva Estatal de Dzilam, Yucatán. Fecha de publicación 29 septiembre de 2006.

Halfter, G., y C. E. Moreno. 2005. Significado biológico de las diversidades alfa, beta y gama. Pp. 5-18 in Sobre diversidad biológica: El significado de las diversidades (Halfter, G., J. Soberón, P. Koleff, y E. Melic, eds.). Monografías Tercer Milenio, Vol. 4. S.E.A., CONABIO, CONACYT, DIVERSITAS.

Hernández-Betancourt, S. F., V. Sánchez-Cordero, J. E. Sosa-Escalante, y A. Segovia. 1996. VIII Listados faunísticos de México. Instituto de Biología. UNAM. México, D. F.

Hernández-Betancourt, S. F., J. A. Cimé-Pool, S. Medina Peralta, y M. L. GonzálezVillanueva. 2008a. Fluctuación poblacional de Ototylomys phyllotis Merriam, 1901 (Rodentia: Muridae) en una selva mediana subcaducifolia del sur de Yucatán, México. Acta Zoológica Mexicana (n. s.) 22:161-177.

Hernández-Betancourt, S. F., J. A. Cimé-Pool, y S. Medina Peralta. 2008b. Ecología poblacional de Heteromys gaumeri en la selva del sur deYucatán, México. Pp. 427448 in Avances en el estudio de los mamíferos de México II (Lorenzo Monterrubio, C., E. Medinilla, y J. Ortega, eds.). Asociación Mexicana de Mastozoología, A. C.

Hortal, J., P. A. V. Borges, y C. Gaspar. 2006. Evaluating the performance of species richness estimators sensitivity to simple grain size. Journal of Animal Ecology 75:274-287.

Horváth, A., I. J. March, y J. H. D. Wolf. 2001. Rodent diversity and land use in Montebello, Chiapas, Mexico. Studies on Neotropical Fauna and Environment 36:169-176.

Jones, J. K., H. H. Genoways, y T. E. Lawlor. 1974. Annotated checklist of mammals of the Yucatan Peninsula, Mexico. II. Rodentia. Occasional Papers, The Museum Texas Tech University 2:1-23.

KreBs, C. J. 1985. Ecología. Estudio de distribución y la abundancia. 2ª Ed. Harla. México.

Lawlor, T. E. 1982. Ototylomys phyllotis. Mammalian Species 181:1-3.

Leirana-Alcocer, J. L., S. F. Hernández-Betancourt, L. Salinas-Pebá, y L. L. Guerrero- 
González. 2009. Cambios en la estructura y composición de la vegetación relacionados con los años de abandono de tierras agropecuarias en la selva baja de la Reserva de Dzilam, Yucatán. Polibotanica 27:53-70.

Magurran, A. E. 2004. Measuring biological diversity. Blackwell Publishing. USA.

Medellín, R. A., M. Equinua, y M. A. Amin. 2000. Bat diversity and abundance as indicators of disturbance in Neotropical rainforest. Conservation Biology 14:1666-1675.

Meluink, E. 1985. Agricultural disturbance and rodents: three farming systems in the Sonoran Desert. Journal of Arid Environments 8:207-222.

Meluink, E. 1991. Rodent communities associated with three traditional agroecosystems in the San Luis Potosi Plateau, Mexico. Agriculture, Ecosystems and Environment 33:363-375.

Melunnk, E. 1995. Uso de hábitat, dinámica poblacional y estacional reproductiva de roedores en el Altiplano Potosino, México. Revista Mexicana de Mastozoología $1: 1-8$.

Meneses, R. E. 2008. Fluctuación poblacional de los pequeños roedores en el Parque Nacional de Dzibilchaltún. De septiembre 2006 a enero de 2008. Tesis de Licenciatura. Campus de Ciencias Bilógicas y Agropecuarias. Universidad Autónoma de Yucatán.

MirandA, I.Y. 2010. Riqueza y abundancia de ratones silvestres en cinco áreas naturales protegidas y una zona del sur del estado de Yucatán, México. Tesis de licenciatura. FMVZ-UADY

Moreno, C. E. 2000. Métodos para medir la biodiversidad. UNESCO Sociedad Entomológica Aragonesa. CYTED. España.

Nupp, T. E., Y R. K. Swihart. 1998. Effect of forest fragmentation of population attributes of white-footed mice and eastern chipmunks. Journal of Mammalogy 79:1234-1243.

Ortíz-Díaz, J. J., J. Tún-Garrido, J. S. Flores, M. I. Peraza-Arcila, G. A. Palma-Pech, y D. S. Escárraga-Paredes. 2008. Diversidad florística de las selvas caducifolias de las Áreas Protegidas Estatales de Yucatán, México. Mesoamericana, Noviembre (2008), Revista Oficial de la Sociedad Mesoamericana para la Biología y la Conservación 12:61.

Pozo, C., Y J. E. Escobedo. 1999. Mamíferos terrestres de la reserva de la Biósfera de Sian Ka'an, Quintana Roo, México. Revista Biología Tropical 47:251-262.

ReID, A. F. 2007. A field guide to the mammals of Central America and Southeast Mexico. Oxford University Press. New York. USA.

Rıjas-López, M. E. 2006. Rodent communities in two natural and one cultivated "nopaleras" (Opuntia spp.) in north-eastern Jalisco, Mexico. Journal of Arid Environments 67:428-435.

Rudran, R. 1996. Method for marking mammals. Pp. 299-310 in Measuring and monitoring biological diversity: Standard methods for mammals (Wilson, D. E., F. R. Cole, J. D. Nochols, R. Rudran, y M.S. Foster, eds.). Smithsonian Institution Press. Washington, D. C.

SáeNz, J.C. 1999. Movimientos y selección de microhábitat de una rata arborícola Ototylomys phyllotis (Rodentia: Muridae) en un bosque seco tropical. Brenesia 52:61-64.

Sánchez-Cordero, V., A. T. Peterson, E. Martínez-Meyer, y R. Flores. 2005. Distribución de roedores reservorios del virus causante del síndrome pulmonar por hantavirus y 
regiones de posible riesgo. Acta Zoológica Mexicana (n. s.) 21:79-92.

Schmidt, C. A., M. D. Engstrom, y H. H. Genoways. 1989. Heteromys gaumeri. Mammalian Species 345:1-4.

Schnell, G. D., M. L. Kennedy, C. Sánchez-Hernández, M. L. Romero-Almaraz, B. D. N. Estevez, J. A. Guerrero, T. L. Best, M. C. Wooten, y R. D. Owen. 2008. Habitat preference of the endemic tawny deermouse (Peromyscus perfulvus), a species of conservation concern. The Southwestern Naturalist 53:9-20.

Tøttrup, A. P., F. P. Jensen, y K. D. Christensen. 2005. The avifauna of two woodlands in Southeast Tanzania. Scopus 25:23-36.

Vera y Conde, C. F., y C. F. D. Rocha. 2006. Habitat disturbance and small mammal richness and diversity in an Atlantic rainforest area in southeastern Brazil. Brazilian Journal of Biology 66:983-990.

Young, C. J., Y J. K. Jones. 1983. Peromyscus yucatanicus. Mammalian Species 196:1-3.

YounG, C. J., Y J. K. Jones. 1984. Reithrodontomys gracilis. Mammalian Species 218:1-3.

ZAR, J. H. 1999. Biostatistical analysis. Fourth Ed. Prentice-Hall. New Jersey, USA.

Sometido: 19 octubre 2009

Revisado: 13 enero 2010

Aceptado: 23 febrero 2010

Editor asociado Consuelo Lorenzo 
